\title{
Anatomía Foliar de Calliandra riparia Pittier en un Gradiente Altitudinal de la Cuenca del Río Tocuyo, Lara, Venezuela
}

Foliar Anatomy of Calliandra riparia Pittier an Elevation Gradient on the High Basin of the Tocuyo River, Venezuela

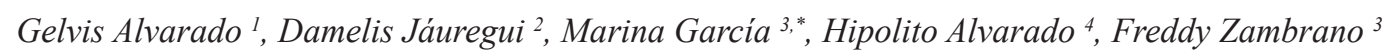

INFORMACIÓN DEL

ARTÍCULO

Fecha de recepción: 30 de abril de 2019.

Fecha de aceptación: 12 de junio de 2019.

${ }^{1}$ Universidad Pedagógica Experimental Libertador, Laboratorio de Microscopía Óptica Dr. Fulgencio Proverbio, Caracas-Venezuela.

2 Universidad Central de Venezuela, Facultad de Agronomía, Instituto de Botánica Agrícola,

3 Universidad Técnica de Manabí, Facultad de Ingeniería Agronómica, Portoviejo-Ecuador.

${ }^{4}$ Universidad Centroccidental Lisandro Alvarado, Decanato de Agronomía, Lara-Venezuela.

* Autor de correspondencia: Marina García, 3Universidad Técnica de Manabí, Facultad de Ingeniería Agronómica, Portoviejo-Ecuador. E-mail: marinabotanica@gmail.com. Tlf: (593-9) 99614935

ENLACE DOI: http://dx.doi.org/10.31095/investigatio. 2020.13.2

\section{Resumen}

Se estudió la anatomía foliar de Calliandra riparia en un gradiente altitudinal con el fin de establecer la posible plasticidad estructural de esta especie para adaptarse a los cambios ambientales relacionados con el aumento en la altitud. Se recolectaron hojas a 800 y $1030 \mathrm{msnm}$, se fijaron en FAA (formaldehido, ácido acético y etanol) y luego se procesaron usando técnicas clásicas utilizadas en microscopía óptica. La anatomía de la lámina, de la nervadura central y del pecíolo fue similar en las dos altitudes consideradas; sin embargo, se observaron diferencias en las variables anatómicas cuantificadas a nivel de lámina, las cuales parecen estar relacionadas con el aumento en la radiación solar a mayor altitud. Dichas diferencias son evidencia de que esta especie desarrolla algunas estrategias anatómicas a nivel foliar para su adaptación a lo largo del gradiente altitudinal.

\section{Palabras Clave:}

Altitud; estructura anatómica; Calliandra; foliólulos; peciolo.

\section{Clasificación JEL: Q2.}

\begin{abstract}
The leaf anatomy of Calliandra riparia was studied in an altitudinal gradient in order to establish the possible structural plasticity of this species to cope with environmental changes related with the increase on altitude. Leaves of adult plants growing at 800 and 1030 masl were collected, fixed in FAA (formaldehyde, acetic acid and ethanol) and then processed by classical techniques used in optical microscopy. The anatomy of the lamina, the midrib and the petiole were similar at both considered altitudes; however, differences were observed on quantitative anatomical variables of the leaf blade which seem to be related to the increase in solar radiation at the higher altitude. These differences show that this species develops some anatomical strategies at foliar level for its adaptation along the altitudinal gradient.
\end{abstract}

Keywords:

Altitude; anatomical structure; Calliandra; foliole; petiole.

JEL Classification: Q2.

13

INVESTIGATIO No. 13, marzo 2020, pp. 13-24,

ISSN: 1390 - 6399・ISSN-e: 2602 - 8336 


\section{Introducción}

Los estudios de aclimatación en plantas son importantes en la comprensión de las modificaciones adaptativas que se producen en ellas, debido a la variación en las condiciones ambientales. Se sabe que cambios en el gradiente altitudinal están asociados con modificaciones en la temperatura, humedad, intensidad luminosa, radiación, exposición al viento, fertilidad del suelo, entre otros. La combinación de todos estos factores ejerce presión sobre las plantas, la cual se expresa como cambios no solo en su fisiología, sino también en su morfología y anatomía (Kofidis et al., 2003; Molina-Montenegro y Cavieres, 2010; Cornelius et al., 2013).

El estudio de los cambios morfo-anatómicos de la hoja para distintos gradientes de altitud ha producido respuestas controversiales en diferentes especies (Carlquist, 1994; Kofidis et al., 2003; Ely et al., 2005; Akinlabi et al., 2014; Jiménez-Noriega et al., 2015). Carlquist (1994) comentó sobre la importancia de tener un mayor número de trabajos que evalúen los cambios morfo-anatómicos de las especies a lo largo de gradientes altitudinales para precisar las adaptaciones que pueden existir bajo condiciones cambiantes con la altitud.

Las especies de Calliandra Benth., en general, no han sido estudiadas en detalle desde el punto de vista de su aprovechamiento económico; no obstante, las integrantes de dicho género presentan características tales como: crecimiento rápido, capacidad de colonizar suelos pobres y arquitectura vegetativa $y$ de sus inflorescencias, atractiva, que lo hacen una fuente potencial de uso importante. Uno de los aspectos económicos más relevante es el ornamental, el cual no ha sido explotado significativamente (Bello y Forero, 2005). Es necesario acotar que la información morfológica (sensu lato) de órganos vegetativos $\mathrm{y}$ reproductivos disponible para este género es escasa, pudiendo citarse: la descripción morfoanatómica de la cubierta seminal de cinco especies de Calliandra, incluida $C$. riparia (Leython y Jáuregui, 2008); los estudios sobre la madera de $C$. laxa (Willd.) Benth. (León, 2008) y acerca del enraizamiento de estacas de $C$. brevipes Benth. y C. tweedii Benth. (Mayer, Cardoso et al., 2008). En el caso de anatomía foliar, Lersten y Curtis (1993) hicieron una descripción detallada del mesofilo paravenal en $C$. tweedii y $C$. emarginata (Humb. \& Bonpl. ex Willd.) Benth. El-Emary et al. (2003) caracterizaron macro y micromorfológicamente los tallos y las hojas de C. haematocephalla Hassk. y por último, Serra-Gama et al. (2009), quienes realizaron la caracterización anatómica foliar de $C$. surinamensis Benth.

Calliandra riparia Pittier es una leguminosa del grupo de las Mimosoideae (Judd et al., 2008), que fue descrita por primera vez por Henri Pittier en Venezuela (Pittier, 1927). Sin embargo, crece de forma natural desde Panamá hasta Brasil. No es muy referida en la literatura, a pesar de ser una especie dominante en regiones secas del norte de 
América del Sur; además de ser cultivada ampliamente tanto en su área de distribución nativa como en otros lugares (Bello y Forero, 2005). En Venezuela, esta especie crece en márgenes de ríos, a una altitud que varía entre 50 y $1200 \mathrm{msnm}$ (Hokche et al., 2008), lo cual hace presumir que la misma debe poseer cierto grado de plasticidad para poder adaptarse a los cambios ambientales que ocurren en ese rango de altitud.

En esta investigación se estudió la anatomía foliar en especímenes de $C$. riparia creciendo en dos sectores de la cuenca alta del río Tocuyo (Lara, Venezuela), ubicados a dos niveles altitudinales diferentes con el objetivo de evaluar las posibles estrategias estructurales en este órgano, que permiten la adaptación de esta especie al incremento en la altitud.

\section{Materiales y Métodos}

El estudio se realizó en dos sectores de la cuenca alta del río Tocuyo, Municipio Morán del estado Lara-Venezuela, a 800 y $1030 \mathrm{msnm}$. En cada sector, se registraron datos de temperatura y humedad relativa, con un termohigrómetro marca HANNA Instruments, modelo H18564; también se midió la intensidad lumínica a través de un radiómetro marca ACCUPAR, modelo LP-80; los valores promedio de estas variables se muestran en la Tabla 1. Así mismo, en cada sitio de muestreo se tomó una muestra de suelo para el análisis de textura, $\mathrm{pH}$ y conductividad eléctrica (CE). La textura de los suelos fue franco-arenosa en los dos sectores; mientras que el $\mathrm{pH}$ de ambos resultó moderadamente ácido; y la conductividad eléctrica (CE) fue similar e inferior a 1 dS. $\mathrm{m}^{-1}$ en los dos sitios de muestreo, lo cual significa que se trata de suelos no salinos.

Tabla 1.

Valores promedio de las variables climáticas medidas en dos sectores de la cuenca alta del río Tocuyo

\begin{tabular}{lccc}
\hline \multicolumn{1}{c}{ Sector } & $\begin{array}{c}\text { Radiación } \\
(\boldsymbol{\mu m o l . ~ m - 2 . s - 1 )})\end{array}$ & $\begin{array}{c}\text { Humedad } \\
\text { Relativa (\%) }\end{array}$ & $\begin{array}{c}\text { Temperatura } \\
\left({ }^{\mathbf{O}} \mathbf{C}\right)\end{array}$ \\
\hline $\begin{array}{l}\text { La Esperanza } \\
(800 \text { msnm) }\end{array}$ & 1924 & 60 & 31 \\
$\begin{array}{l}\text { La Palomera } \\
(1030 \mathrm{msnm})\end{array}$ & 2265 & 60 & 29 \\
\hline
\end{tabular}

En cada sector, se seleccionaron aleatoriamente tres individuos adultos de C. riparia y en cada uno de ellos, se muestreó un total de seis hojas maduras ubicadas entre el quinto y sexto nudo en sentido ápice-base de ramas expuestas. Se tomó la porción media de foliólulos ubicados en la parte central de las hojas. Las muestras se lavaron con agua y se fijaron en FAA (formaldehído-ácido acético-etanol 70\%) hasta su procesamiento. Posteriormente, el material fue deshidratado en una batería ascendente de alcohol butílico terciario (ABT) y luego se incluyó en parafina para efectuar secciones transversales, con un micrótomo de rotación. Se hizo tinción doble progresiva con safranina-fast green y el montaje se realizó en bálsamo de Canadá para la obtención de láminas permanentes (Johansen, 1940). Adicionalmente, se realizaron cortes transversales a mano alzada para efectuar pruebas histoquímicas con el fin de detectar almidón y taninos, siguiendo los protocolos propuestos por Johansen (1940). Para el estudio de las epidermis se 
hicieron macerados parciales con hipoclorito de sodio comercial al 3,25\% (Sandoval, 2005), las porciones obtenidas se tiñeron con azul de toluidina acuosa $(0,1 \%)$ y se montaron en una solución de agua- glicerina (v:v).

Las preparaciones microscópicas fueron observadas bajo un microscopio óptico Nikon E-200 y se tomaron imágenes digitales con una cámara marca Evolution LC Color incorporada al microscopio. Asimismo, con ayuda de un ocular micrométrico se midió: grosor de epidermis (adaxial y abaxial), espesor del parénquima en empalizada, parénquima esponjoso y de la lámina; también se contaron estomas y tricomas, para determinar la densidad de dichas estructuras. Se efectuaron 30 mediciones para cada variable, en distintas láminas.

Los datos de las variables anatómicas cuantitativas se sometieron a un análisis de varianza (ANOVA), comparando entre los pisos altitudinales, y en los casos en que se

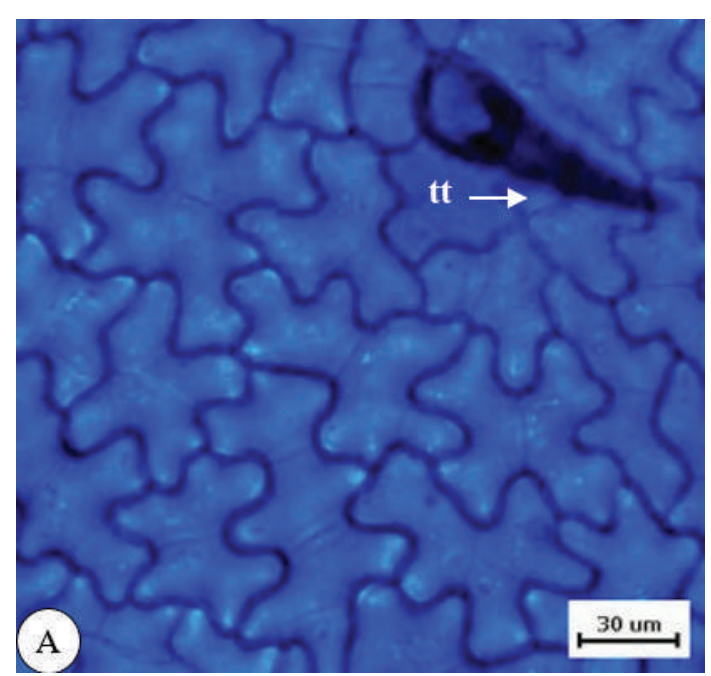

detectó diferencias estadísticas significativas se realizó comparación de medias mediante la prueba de Tukey al nivel de significancia del 5\%. El software utilizado fue el InfoStat (Di Rienzo et al., 2014).

\section{Resultados y Discusión}

La anatomía de la lámina, de la nervadura central y del pecíolo de $C$. riparia fue similar en las dos altitudes consideradas; sin embargo, se observaron diferencias en las variables cuantificadas a nivel de lámina foliar.

\section{Lámina foliar}

En vista frontal, las células epidérmicas típicas presentan paredes onduladas y relativamente delgadas, tanto en la epidermis adaxial (Figura 1A) como en la abaxial (Figura 1B). Se observaron dos tipos de tricomas: 1) tricomas tectores unicelulares de ápice agudo, de longitud variable, ubicados en ambas epidermis (Figura 1); y 2) tricomas

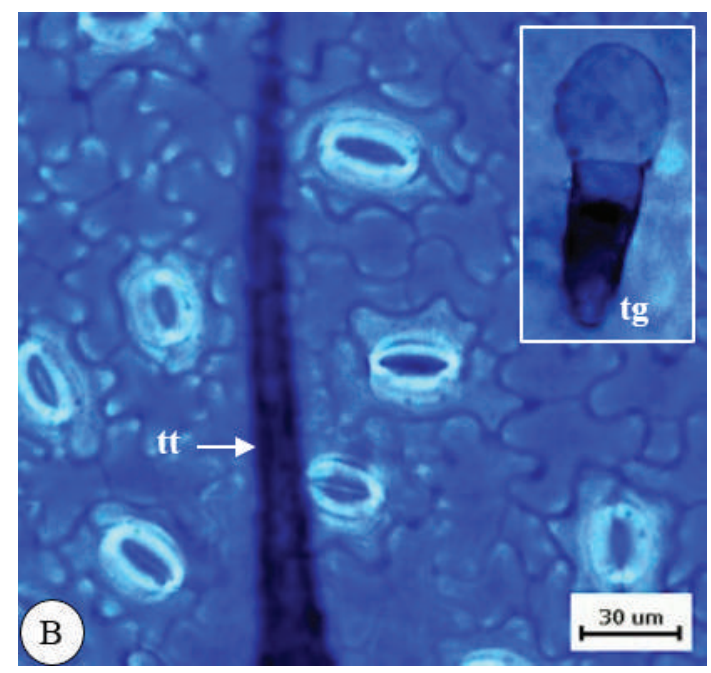

Figura 1. Vistas paradérmicas de las epidermis foliares de Calliandra riparia, a $1030 \mathrm{msnm}$, adaxial (A) y abaxial (B). tg: tricoma glandular; tt: tricoma tector. 
glandulares con pedicelo bicelular y cabeza unicelular, solo en la superficie abaxial (Figura 1B). Hojas anfiestomáticas con estomas anomocíticos (Figura 1B) pero en la epidermis adaxial, éstos solo se localizan en la proximidad de las venas. En sección transversal, las epidermis son uniestratificadas, con células cuadrangulares a rectangulares en ambas superficies, la mayoría de ellas con taninos (Figura 2).

La hoja es bifacial (Figura 2), con el mesofilo formado por una o dos capas de parénquima en empalizada, de células alargadas y estrechas, y por parénquima esponjoso que presenta de tres a cuatro capas de células, con pocos espacios intercelulares. Los haces vasculares son de tipo colateral cerrado, los mayores están rodeados por un anillo esclerenquimático; mientras que los haces menores tienen esclerénquima, adaxial y abaxialmente. Se observaron cristales

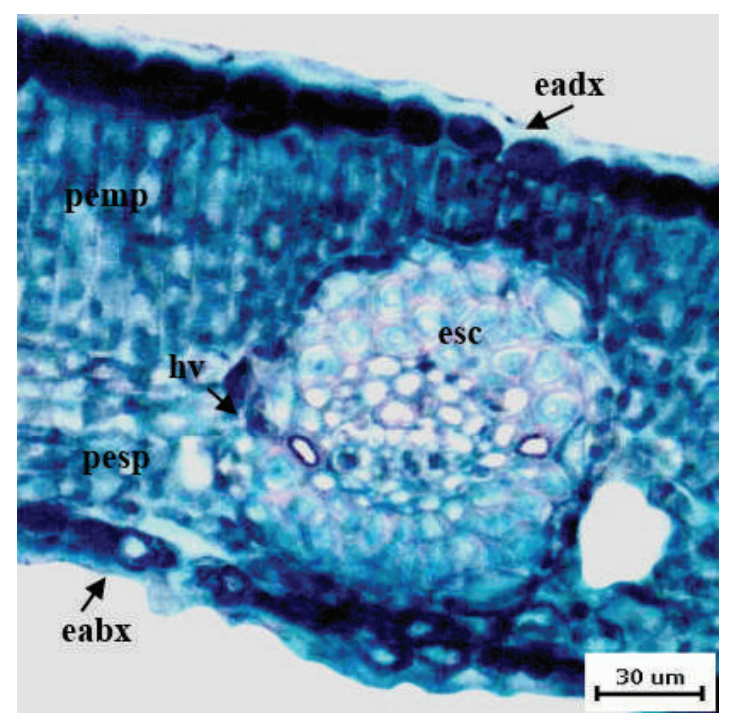

Figura 2. Sección transversal de la lámina foliar de Calliandra riparia, a $800 \mathrm{msnm}$. eadx: epidermis adaxial; eabx: epidermis abaxial; esc: esclerénquima; hv: haz vascular; pemp: parénquima en empalizada; pesp: parénquima esponjoso. romboédricos en las células parenquimáticas adyacentes a la vaina esclerenquimática de los haces vasculares.

La nervadura central (Figura 3) posee epidermis uniestratificada; las células epidérmicas adaxiales son similares a las de la lámina, pero las de la epidermis abaxial tienen pared celular externa convexa; las células de ambas epidermis contienen taninos. Debajo de la epidermis adaxial, es evidente una capa de parénquima clorofiliano con células de forma similar a las del parénquima en empalizada de la lámina, pero mucho más cortas, mientras que en la superficie abaxial se distinguen dos capas de tejido parenquimático de células redondeadas. La porción central de la nervadura está ocupada por el tejido vascular, el cual está constituido por un haz colateral cerrado, delimitado por un anillo esclerenquimático y por fuera de éste se diferencia una vaina parenquimática con abundantes cristales romboédricos.

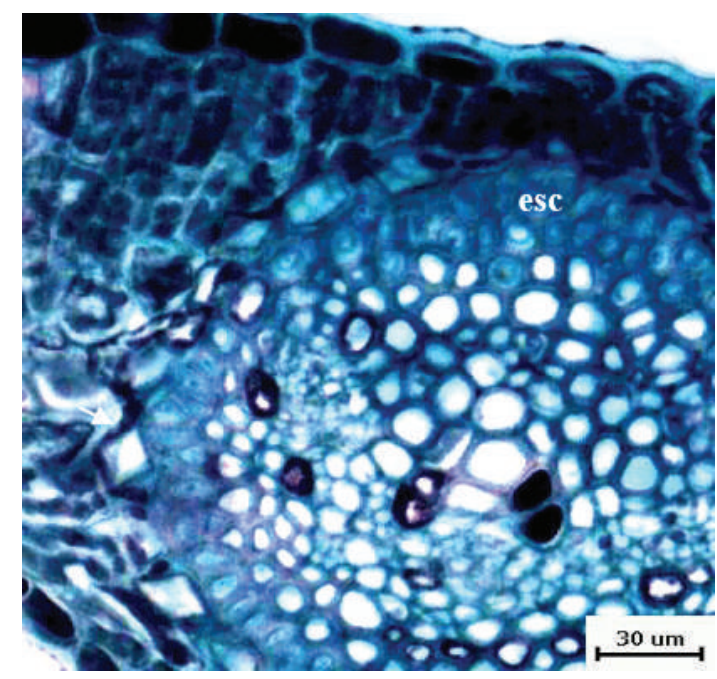

Figura 3. Sección transversal de la nervadura central de Calliandra riparia, a $800 \mathrm{msnm}$. esc: esclerénquima. 
Pecíolo (Figura 4): es de contorno aproximadamente circular, con un saliente a cada lado hacia la superficie adaxial. Epidermis uniestratificada, de células pequeñas, redondeadas, con la pared externa más cutícula muy gruesa y con taninos; solo se observaron tricomas tectores unicelulares alargados, similares a los descritos en la lámina. La región cortical presenta de cuatro a cinco capas de tejido clorenquimático, con taninos y granos de almidón en algunas células. Asimismo, en esta región, son evidentes cristales prismáticos, los cuales son abundantes en la capa adyacente al tejido esclerenquimático que bordea al tejido vascular. El sistema vascular está constituido por tres haces vasculares colaterales abiertos que adoptan forma de arco, ubicados hacia la superficie abaxial y uno prominente también colateral abierto hacia el lado adaxial, estos haces presentan fibras organizadas a modo de casquetes, y con intrusiones hacia el parénquima que separa cada haz en la superficie abaxial; adicionalmente, en

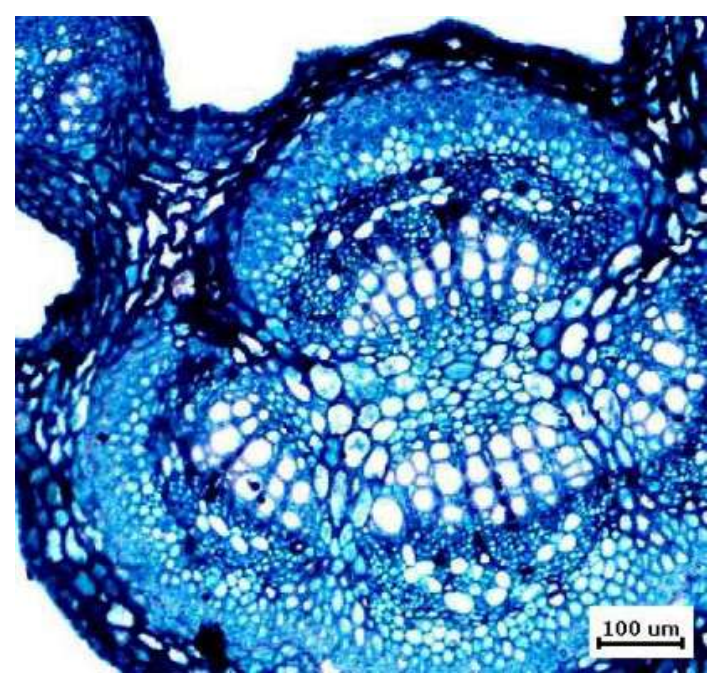

Figura 4. Sección transversal del pecíolo de Calliandra riparia, a $800 \mathrm{msn}$. cada saliente se observó un haz vascular de tipo colateral cerrado, con un casquete de fibras en la región floemática.

Algunas de las características señaladas para $C$. riparia coinciden con lo expuesto por Metcalfe y Chalk (1950) para el género Calliandra; sin embargo, en esta especie no se observaron papilas ni sílice. Asimismo, hay similitud con $C$. surinamensis (Serra-Gama et al., 2009), en el tipo de hoja de acuerdo a la ubicación de los estomas y en el tipo de éstos. Por otra parte, comparte también caracteres estructurales con los referidos para la hoja de $C$. haematocephala (El-Emary et al., 2003); no obstante, estos taxones se diferencian en el tipo de estomas, tipo de hoja de acuerdo al arreglo del mesofilo, el arreglo histológico de la nervadura central y el número y disposición de los haces vasculares presentes en el pecíolo. Se debe destacar además, que en $C$. riparia no se observó mesofilo paravenal, tejido descrito por Lersten y Curtis (1993) en dos especies de Calliandra. Las diferencias anatómicas entre estas especies reafirman la importancia diagnóstica que desde hace mucho tiempo se ha asignado a la anatomía en la delimitación taxonómica de distintos grupos de angiospermas, en este caso a nivel específico.

De los caracteres descritos, algunos se consideran adaptación a condiciones ambientales particulares, pudiendo destacar, la abundancia de taninos, que en el caso de $C$. riparia, se localizan principalmente en sus epidermis y en el parénquima del pecíolo; sin embargo, es 
necesario realizar un análisis cuantitativo para saber si el contenido incrementa con la altitud. Estos compuestos pueden variar con las condiciones ambientales (Close y McArthur, 2002), y pueden actuar como barrera para la penetración excesiva de luz ultravioleta, como ha sido referido en Andira legalis (Vell) Toledo (Carvalho, Barros y Scarano, 2009). Otra variable que amerita un análisis cuantitativo es la frecuencia de cristales en el tejido mesofilar, ya que su presencia puede estar ligada a la genética de la especie, pero en ocasiones puede estar influenciada por factores externos (Molano-Flores, 2001; Borrelli et al., 2016).

El efecto de la altitud sobre variables anatómicas cuantitativas se muestra en la Tabla 2. El grosor de la pared celular más cutícula abaxial y el espesor de la lámina del foliólulo no mostraron diferencias estadísticamente significativas en los valores obtenidos en las dos altitudes consideradas; sin embargo, se debe destacar que se produjo un incremento significativo $(p \leq 0,05)$ en el espesor del parénquima en empalizada a expensas del decremento en el grosor del parénquima esponjoso, por lo que no se produjo una variación significativa en esta última variable, pero sí se evidenció un aumento en la relación parénquima en empalizada/parénquima esponjoso. Dicho incremento puede estar vinculado con el aumento en la intensidad lumínica con la altitud (Tabla 1), ya que se ha señalado que el parénquima en empalizada es muy sensible a los cambios en esta variable climática, debido a su importante papel en la captura de luz (Tsukaya, 2005).

El aumento significativo $(p \leq 0,05)$ en el grosor de la pared celular más cutícula adaxial puede servir de mecanismo de protección contra la excesiva radiación, ya que esta última es capaz de generar calentamiento excesivo de los tejidos internos y en consecuencia daño de los

Tabla 1.

Valores promedio y desviación estándar de las variables anatómicas cuantitativas determinadas en foliólulos de Calliandra riparia, creciendo a dos altitudes

\begin{tabular}{lcr}
\hline \multirow{2}{*}{ VARIABLES } & \multicolumn{2}{c}{ ALTITUD (msnm) } \\
\cline { 2 - 3 } & $\mathbf{8 0 0}$ & $\mathbf{1 0 3 0}$ \\
\hline Grosor pared celular más cutícula adaxial $(\mu \mathrm{m})$ & $2,69 \pm 0,01 \mathrm{~b}$ & $3,01 \pm 0,06 \mathrm{a}$ \\
Grosor pared celular más cutícula abaxial $(\mu \mathrm{m})$ & $1,98 \pm 0,06 \mathrm{a}$ & $2,02 \pm 0,01 \mathrm{a}$ \\
Grosor epidermis adaxial $(\mu \mathrm{m})$ & $14,18 \pm 0,20 \mathrm{a}$ & $11,52 \pm 0,86 \mathrm{~b}$ \\
Grosor epidermis abaxial $(\mu \mathrm{m})$ & $7,74 \pm 0,11 \mathrm{a}$ & $6,62 \pm 0,25 \mathrm{~b}$ \\
Grosor parénquima en empalizada $(\mu \mathrm{m})$ & $53,76 \pm 1,99 \mathrm{~b}$ & $61,81 \pm 0,28 \mathrm{a}$ \\
Grosor parénquima esponjoso $(\mu \mathrm{m})$ & $34,08 \pm 3,32 \mathrm{a}$ & $26,92 \pm 0,99 \mathrm{~b}$ \\
Relación P. en empalizada/P. esponjoso & $1,62 \pm 0,15 \mathrm{~b}$ & $2,31 \pm 0,07 \mathrm{a}$ \\
Grosor lámina $(\mu \mathrm{m})$ & $114,43 \pm 4,59 \mathrm{a}$ & $111,90 \pm 0,90 \mathrm{a}$ \\
Densidad de estomas adaxial $\left(\mathrm{N}^{\circ}\right.$ estomas $\left./ \mathrm{mm} 2\right)$ & $8,81 \pm 0,76 \mathrm{~b}$ & $21,67 \pm 2,17 \mathrm{a}$ \\
Densidad de estomas abaxial $\left(\mathrm{N}^{\circ}\right.$ estomas $\left./ \mathrm{mm} 2\right)$ & $404,52 \pm 26,51 \mathrm{~b}$ & $440,24 \pm 6,64 \mathrm{a}$ \\
Densidad de tricomas adaxial $\left(\mathrm{N}^{\circ}\right.$ tricomas $\left./ \mathrm{mm} 2\right)$ & $5,95 \pm 0,41 \mathrm{~b}$ & $45,00 \pm 0,71 \mathrm{a}$ \\
Densidad de tricomas abaxial $\left(\mathrm{N}^{\circ}\right.$ tricomas $\left./ \mathrm{mm} 2\right)$ & $7,14 \pm 2,14 \mathrm{~b}$ & $27,86 \pm 1,24 \mathrm{a}$ \\
Índice estomático adaxial & $0,55 \pm 0,08 \mathrm{~b}$ & $2,08 \pm 0,12 \mathrm{a}$ \\
Índice estomático abaxial & $18,95 \pm 0,76 \mathrm{~b}$ & $26,00 \pm 1,07 \mathrm{a}$ \\
\hline
\end{tabular}

Nota: Medias con una letra común en la misma fila no son significativamente diferentes para el test de Tukey $(\mathrm{p} \leq 0,05)$. 
mismos; se le atribuye adicionalmente, una acción mecánica al prevenir el colapso de la hoja cuando pierde mucha agua (Jacobs et al., 2007; Nejadhabibvash et al., 2017). Por otra parte, el espesor de las células epidérmicas disminuyó significativamente $(\mathrm{p} \leq 0,05)$ con la altitud; respuesta similar fue observada por Jiménez-Noriega et al. (2015) en Ribes ciliatum creciendo en un gradiente altitudinal, pero no discuten al respecto. Se ha indicado que la radiación UV-B, la cual es comúnmente superior a mayor altitud, ejerce un efecto inhibitorio sobre la expansión de las células epidérmicas en la cara adaxial, que es la que principalmente recibe dicha radiación (Carrasco-Ríos, 2009).

Los valores de densidad de tricomas fueron mayores a $1030 \mathrm{msnm} y$ significativamente diferentes $(\mathrm{p} \leq 0,05)$ de los determinados a $800 \mathrm{msnm}$. Se ha indicado que el incremento en la densidad de tricomas a medida que aumenta la altitud, supone una estrategia importante para proteger los tejidos foliares contra la radiación intensa y la acción de los vientos, contribuyendo además a aumentar la reflectancia de la luz, con lo cual se impide el sobrecalentamiento de la hoja evitando así la transpiración excesiva (Levizou et al., 2004; Molina-Montenegro, 2008); sin embargo, una tendencia contraria, también ha sido reportada por Jiménez-Noriega et al., (2015) quienes indicaron que probablemente hubo un efecto compensatorio, ya que dicha función pudo ser asumida por el aumento en el contenido de ciertos pigmentos (Molina-Montenegro, 2008).
El aumento en el número de estomas por unidad de área a medida que se incrementa la altitud, también fue significativo $(\mathrm{p} \leq 0,05) \quad y \quad$ ha sido observado en otros estudios donde vinculan rasgos anatómicos con altura sobre el nivel del mar, entre ellas el de Paridari, Jalali, Zarafshar y Bruschi, (2013) en Carpinus betulus L.; esta respuesta se ha asociado con mejoras en la eficiencia para tomar dióxido de carbono (McElwain, 2004). Hay investigadores que indican que el incremento en la densidad estomática obedece a un aumento en la intercepción de radiación solar con la altitud, ejerciendo la intensidad de la luz un efecto positivo sobre esa variable (Körner, 1999 citado por Wang et al., 2014). No obstante, hay especies donde la respuesta al incrementar la altitud es contraria, es decir, ocurre una disminución en la densidad estomática, lo cual se ha atribuido al efecto de una menor concentración de $\mathrm{CO}_{2}$ en sitios más elevados, por la influencia que ejerce esta molécula sobre la diferenciación de estomas; una respuesta como ésta se reportó en Chromolaena odorata (L.) King \& Robinson (Akinlabi et al., 2014).

El comportamiento del índice estomático fue similar al ya descrito para la densidad de estomas; no obstante, los resultados de otros estudios han mostrado una tendencia controversial de esta variable, entre ellos el de Royer (2001), ya que en ocasiones disminuye, como ocurrió en $R$. ciliatum (Jiménez-Noriega et al., 2015), mientras que en otros casos aumenta; Kouwenberg et al. (2007), trabajando con Quercus kelloggii Newb. 
y Nothofagus solandri var. cliffortioides (Dippel) Poole señalaron que tanto la densidad como el índice estomático aumentaron con la altitud, indicando que los principales factores que modifican esta última variable son los niveles de dióxido de carbono y la intensidad de luz; sin embargo, indican que otros factores pueden modular esta respuesta.

\section{Conclusiones}

En conclusión, C. riparia mostró cambios estructurales en características anatómicas cuantitativas inducidos por las presiones ambientales impuestas por la variación de altitud, probablemente con una influencia marcada de la radiación solar. Dichos cambios son evidencia de que esta especie presenta algunas estrategias a nivel foliar para desarrollarse a lo largo de un gradiente altitudinal.

\section{Referencias}

Akinlabi, A.A., Jimoh, M.A. y Saheed, S.A. (2014). Effects of altitudinal gradients on morpho anatomical characters of Chromolaena odorata (L.) King \& Robinson. FUTA Journal of Research in Sciences, (2), 150-156.

Bello, M.A., y Forero E. (2005). Revisión del género Calliandra (Leguminosae: Mimosoideae: Colombia. En D.C. Romero y E. Forero (Eds), Estudios en leguminosas colombianas (pp. 39-110) Bogotá, Colombia: Editorial Guadalupe LTDA.

Borrelli, N., Benvenuto M. L. y Osterrieth M. 2016. Calcium oxalate crystal production and density at different phenological stages of soybean plants (Glycine max L.) from the southeast of the Pampean Plain,
Argentina. Plant Biology, 18(6), 1016-1024.

Carlquist S. (1994). Anatomy of tropical alpine plants. En P.W. Rundel, A.P. Smith y F.C. Meinzer (Eds.), Tropical Alpine Enviroments: Plant Form and Function (pp. 111-125), Cambridge: Cambridge University Press.

Carrasco-Ríos L. (2009). Efecto de la radiación ultravioleta-B en plantas. Idesia, 27(3), 59-76.

Carvalho D., Barros C. y Scarano F. (2009). In situ variation in leaf anatomy and morphology of Andira legalis (Leguminosae) in two neighbouring but contrasting light environments in a Brazilian sandy coastal plain. Acta Botanica Brasilica, 23(1), 267-273.

Close, D.C. y McArthur C. (2002). Rethinking the role of many plant phenolics - protection from photodamage not herbivores? OIKOS, 99, 166-172.

Cornelius C., Estrella N., Franz H. y Menzel A. (2013). Linking altitudinal gradients and tempeture responses of plant phenology in the Bavarian Alps. Plant Biology 15 (Supl.), 57-69.

Di Rienzo, J., Casanoves, F., Balzarini, M., Gonzalez, L., Tablada, M. y Robledo, C. (2014) InfoStat versión 2013. Grupo InfoStat, FCA, Universidad Nacional de Córdoba, Argentina. Recuperado de http://www. infostat. com. ar.

El-Emary, N., Makboul, M., Abdel-Hafiz M. y M. Magdy. 2003. Macro-and micromorphology of the stem and leaf of Calliandra haematocephala (Hassk.) cultivated in Egypt. Bulletin Pharmaceutical Sciences, 26, 153-170.

Ely, F., Torres, F. y Gaviria, J. (2005). Relación entre la morfoanatomía foliar de tres especies de Miconia (Melastomataceae) 
con su hábitat y distribución altitudinal en el parque nacional Sierra Nevada de Mérida, Venezuela. Acta Botanica Venezuelica, 28(2), 275-300.

Hokche, O., Berry P.E. y Huber, O. (eds.) (2008). Nuevo Catálogo de la Flora Vascular de Venezuela 1-860. Caracas: Fundación Instituto Botánico de Venezuela.

Jacobs, J.F., Koper G.J.M. y Ursemb W.N.J. (2007). UV protective coatings: A botanical approach. Progress in Organic Coatings, $58,166-171$.

Jiménez-Noriega, M.S., Terrazas, T. y López-Mata L. (2015). Variación morfo-anatómica de Ribes ciliatum a lo largo de un gradiente altitudinal en el norte de la Sierra Nevada, México. Botanical Sciences, 93(1), 1-10.

Johansen, D. (1940). Plant microtechnique. New York: Mc Graw Hill.

Judd, W., Campbell, Ch.S., Kellogg, E.A., Stevens, P.F. y Donoghue, M.J (2008). (ed.). Sunderland, Massachusetts: Sinauer Associates.

Kofidis, G., Bosabaldis, A.M y Moustakas, M. (2003). Contemporary seasonal and altitudinal variations of structural features in Oregano (Origanum vulgare L.). Annals of Botany, 92, 635-645.

Körner C. (1999). Alpine plant life. Berlin: SpringerVerlag.

Kouwenberg, L.L., Kürschner, W.M. y McElwain, J.C. (2007). Stomatal frequency change over altitudinal gradients: prospects for paleoaltimetry. Reviews in Mineralogy $y$ Geochemistry, 66, 215-241.

Kürschner, W., Stulen, I., Wagner, F. y Kuiper, P. (1998) Comparison of palaeobotanical observations with experimental data on the leaf anatomy of durmast oak (Quercus petraea) (Fagaceae) in response to environmental changes. Annals of Botany,
$81,657-664$

León, W. (2008). Anatomía de madera en 31 especies de la subfamilia Mimosoideae (Leguminosae) en Venezuela. Colombia Forestal, 11, 113-135.

Lersten, N.R. y Curtis, J.D. (1993). Paraveinal mesophyll in Calliandra tweedii and $C$. emarginata (Leguminosae; Mimosoideae). American Journal of Botany, 80(5), 561-568.

Levizou, E., Drilias, P., Psaras, G.K. y Manetas, Y. (2004). Nondestructive assessment of leaf chemistry and physiology through spectral reflectance measurements may be misleading when changes in trichome density co-occur. New Phytology, 165, 463-472.

Leython, S. y Jáuregui D. (2008). Morfología de la semilla y anatomía de la cubierta seminal de cinco especies de Calliandra (Leguminosae-Mimosoideae) de Venezuela. Revista Biología Tropical, 56(3), 1075-1086.

Mayer, J.L.S., Cardoso, N.A., Cuquel, F. y Bona C. (2008). Formação de raízes em estacas de duas espécies de Calliandra ( Le guminos a e-Mimosoideae). Rodriguesia, 59(3), 487-495.

McElwain, J.C. (2004). Climate-independent paleoaltimetry using stomatal density in fossil leaves as a proxy for $\mathrm{CO}_{2}$ partial pressure. Geology, 32, 1017-1020.

Metcalfe, C.R y Chalk, L. (1950). Anatomy of the dicotyledons. Vol. I, Oxford: Clarendon Press.

Molano-Flores, B. 2001. Herbivory and calcium concentrations affect calcium oxalate crystal formation in leaves of Sida (Malvaceae). Annals of Botany, 88(3), 387-391. 88.

Molina-Montenegro, M. (2008). Variación de la pubescencia foliar en plantas y sus 
implicaciones funcionales a lo largo de gradientes altitudinales. Ecosistemas, $17(1), 146-154$.

Molina-Montenegro M.A. y Cavieres L.A. (2010). Variación altitudinal de los atributos morfo-fisiológicos en dos especies de plantas alto-andinas y sus implicancias contra la fotoinhibición. Gayana Botánica, 67, 1-11.

Nejadhabibvash, F., Rezaei E.Chi. y Pirzad, A. (2017). Anatomy of Salvia limbata in relation to altitudinal gradient in West Azerbaijan (Iran). International Journal of Horticultural Science and Technology, 4(2), 205-216.

Paridari, I. Ch., S.G. Jalali, A. Zarafshar y P. Bruschi. (2013). Leaf macro and micromorphological altitudinal variability of Carpinus betulus in the Hyrcanian forest (Iran). Journal of Forestry Research, 24(2), 301-307.

Pittier, H. (1927). Árboles y arbustos nuevos de Venezuela 6-8, 80.

Royer, D.L. 2001. Stomatal density and stomatal index as indicators of paleoatmospheric $\mathrm{CO}_{2}$ concentration. Review of Palaeobotany and Palynology, 114, 1-28.

Sandoval, E. (2005). Técnicas aplicadas al estudio de la anatomía vegetal. México: Universidad Nacional Autónoma de México.

Serra- Gama, T., de Souza, Garcia, T.B. y AraújoLucas, F.C. (2009). Morfo-anatomia foliar de Calliandra surinamensis Benth. - (Leguminosae - Mimosoideae). En $60^{\circ}$ Congreso Nacional de Botânica, Brasil.

Tsukaya, H. (2005). Leaf shape: genetic controls and environmental factors. The International Journal of Developmental Biology, 49, 547-555.

Wang, R., Yu, G., He, N., Wang, Q., Xia, F., Zhao,
N., Xu, Z. y Ge, J. (2014). Elevationrelated variation in leaf stomatal traits as a function of plant functional type: Evidence from Changbai Mountain, China. PLoS One, 9(12), e115395. 
